\title{
A compact neutron generator using a field ionization source ${ }^{a)}$
}

Arun Persaud, ${ }^{1, \text { b) }}$ Ole Waldmann, ${ }^{1}$ Rehan Kapadia, ${ }^{2}$ Kuniharu Takei, ${ }^{2}$ Ali Javey, ${ }^{2}$ and Thomas Schenkel ${ }^{1}$

${ }^{1)}$ E.O. Lawrence Berkeley National Laboratory, Berkeley, California 94720, USA

${ }^{2)}$ Department of Electrical Engineering and Computer Sciences, University of California at Berkeley, Berkeley, California 94720, USA

(Dated: 16 December 2011)

Field ionization as a means to create ions for compact and rugged neutron sources is pursued. Arrays of carbon nano-fibers promise the high field-enhancement factors required for efficient field ionization. We report on the fabrication of arrays of field emitters with a density up to $10^{6} \mathrm{tips} / \mathrm{cm}^{2}$ and measure their performance characteristics using electron field emission. The critical issue of uniformity is discussed, as are efforts towards coating the nano-fibers to enhance their lifetime and surface properties.

PACS numbers: 29.25.Dz; 79.70.+q; 81.07.De

\section{INTRODUCTION}

Radioactive sources are used in many industrial applications. For example, in the gas/oil industry radioactive sources are routinely employed to characterize the geology of bore holes. In recent years an effort has been made by the U.S. government to reduce the amount of radioactive material available in order to minimize the risk of malevolent use. ${ }^{1}$ A Radiological Source Replacement Program is in effect which includes an investigation into the replacement of radioactive sources for oil-well logging with neutron generators. ${ }^{2}$ Due to the harsh environment in which these generators are used they must fulfill stringent requirements, i.e., ruggedness, compactness, low power consumption, and a long lifetime.

The experiments reported in this article are motivated by the concept to build a neutron source suitable for oil-well logging utilizing the fusion reaction between deuterium(D) and/or tritium(T). In this approach $\mathrm{D} / \mathrm{T}$ ions are created and accelerated towards a deuterated/tritiated target where the fusion reaction takes place upon collision of an ion with a target atom. The D$\mathrm{T}$ fusion reaction has the highest cross-section and results in monoenergetic $14 \mathrm{MeV}$ neutrons, whereas D-D and T$\mathrm{T}$ reactions have roughly $100 \times$ smaller cross sections. A D-D reaction results in the creation of monoenergetic $2.4 \mathrm{MeV}$ neutrons, whereas with a $\mathrm{T}-\mathrm{T}$ reaction there is a continuous spectrum of neutron energies, very similar to the neutron spectrum generated by the radioactive AmBe sources used in industry today. This similarity has the advantage that new and old data can be easily compared. For oil-well logging applications neutron yields of the order $10^{8} \mathrm{n} / \mathrm{s}$ are required, which for $\mathrm{D}-\mathrm{T}$ reactions translates into an ion current of several tens of $\mu \mathrm{A}$ at acceleration energies of $100 \mathrm{keV}$. The energy dependence of the fusion cross-section is such that a higher velocity

\footnotetext{
a) Contributed paper, published as part of the Proceedings of the 14th International Conference on Ion Sources, Giardini Naxos, Italy, September 2011.

b) Electronic mail: APersaud@lbl.gov
}

leads to a higher neutron yield. ${ }^{3}$ Consequently, it is desirable to accelerate ion beams of high atomic fraction, i.e., $\mathrm{D}$ instead of $\mathrm{D}_{2}$, and to use the highest possible acceleration voltage. However, due to size constraints on the power supply and breakdown issues in a compact device, the acceleration voltage will be limited to around $100 \mathrm{kV}$.

One approach to generate the $\mathrm{D} / \mathrm{T}$ ions required for the fusion reaction is to use a plasma source. ${ }^{4}$ As an alternative we have chosen to develop a setup based on field ionization, since the plasma-free environment offered by field emitters enables a compact source, lower energy usage (no energy required to sustain the plasma), and a long lifetime. In field ionization a high electric field is applied to a target resulting in the ionization of atoms due to tunneling of electrons into the vacuum. In order to create a tunnel barrier low enough for a high probability of ionization, electric fields of the order of $20 \mathrm{~V} / \mathrm{nm}$ are required. In an experimental setup, such fields can be achieved using sharp tips to enhance an externally applied field. Any protrusion (corners, edges, tips) will result in a local field enhancement $\gamma$, where $E_{\text {local }}=\gamma E_{\text {ext }}$. The highest field enhancement can be achieved using the sharpest tips, making carbon nano-fibers (CNFs) highly favorable candidates. The enhancement factor of CNFs can be of the order of several thousands, which reduces the external field needed for field ionization to $20 \mathrm{~V} / \mu \mathrm{m}$ or below.

Field ionization experiments using single tips have reported currents of up to $5 \mathrm{nA} .{ }^{5}$ Thus a parallel array of $10^{6}$ tips should yield the currents needed in our application. One issue with using arrays of tips is that if the tip-to-tip distance becomes too small, the tips will shield each other resulting in a diminished field enhancement factor. It has been reported that a good tip-to-tip distance is roughly twice the height of the tips. ${ }^{6,7}$

In a previous article we reported on results from CNFs grown in a forest-like structure. ${ }^{8}$ Forest samples rely on a small number of tips that extend above the rest and have large field-enhancement factors. However, the properties of these tips and the number of protruding tips per unit area is difficult to control experimentally. Therefore, in this report we focus on results from CNFs grown in a 
patterned array with the goal to minimize shielding effects between tips and to have each tip contributing to the ion current. In order to characterize the tip arrays we measure electron field emission rather than ionization. This has the advantage of allowing for higher currents at lower fields, since electrons can be emitted at fields of $2-4 \mathrm{~V} / \mathrm{nm}$, which is one tenth of that necessary for field ionization. Since the field enhancement factor is purely geometrical it is assumed to be the same for field emission and ionization.

\section{SAMPLES}

The CNF arrays are grown using a plasma-enhanced chemical vapor deposition (PECVD) process. As a catalyst for the CNF growth a $30 \mathrm{~nm}$ nickel layer is used. This layer is patterned in a single lithographic step resulting in $2 \mu \mathrm{m}$ dots with a dot-to-dot spacing of $10 / 20 / 30 \mu \mathrm{m}$ to achieve a near optimal height-spacing ratio. In order to minimize diffusion of the nickel a $30 \mathrm{~nm}$ titanium layer is used beneath it, which was deposited on top of a $200 \mathrm{~nm}$ layer of copper. As the base material a 4 in. silicon wafer with high p-doping and a native oxide layer is used. Each sample has an area of $1 \mathrm{~cm}^{2}$ patterned for CNF growth, resulting in $10^{5}-10^{6}$ tips per sample. The growth takes place at $900^{\circ} \mathrm{C}$ in an $\mathrm{NH}_{3}$ and $\mathrm{C}_{2} \mathrm{H}_{2}$ plasma. During the PECVD process a small number of CNFs grow from each nickel island.

The effect of coating the CNFs with palladium and gold layers of different thicknesses $(5 \mathrm{~nm}-60 \mathrm{~nm})$ was investigated. In order to determine the uniformity of these coatings the samples were imaged using scanning electron microscopy (SEM) and transmission electron microscopy (TEM). Fig. 1 shows a SEM image of a palladium-coated sample with a CNF island spacing of $10 \mu \mathrm{m}$ and a CNF height of around $6 \mu \mathrm{m}$. For the TEM study CNFs were scraped from the silicon sample and then transferred to a TEM grid. A TEM image of the palladium-coated sample is shown in Fig. 2 and reveals a uniform coating. In contrast, CNFs coated with gold showed non-uniform coating. ${ }^{9}$

\section{EXPERIMENTAL SETUP}

Neutron yields from the samples are measured using two electrodes in a parallel plate setup as shown in Fig. 3. One electrode is a titanium target and is either biased to $-80 \mathrm{kV}$ for field ionization experiments or to $100 \mathrm{kV}$ for electron field emission experiments. The other electrode is grounded through a 6485 Keithley picoammeter and the field emitters are attached to this electrode using double-sided copper tape. The distance between the electrodes can also be varied. Care is taken to minimize high-voltage breakdown anywhere in the chamber in order to enable high electric fields between the electrodes. The titanium target can be pre-loaded with deuterium

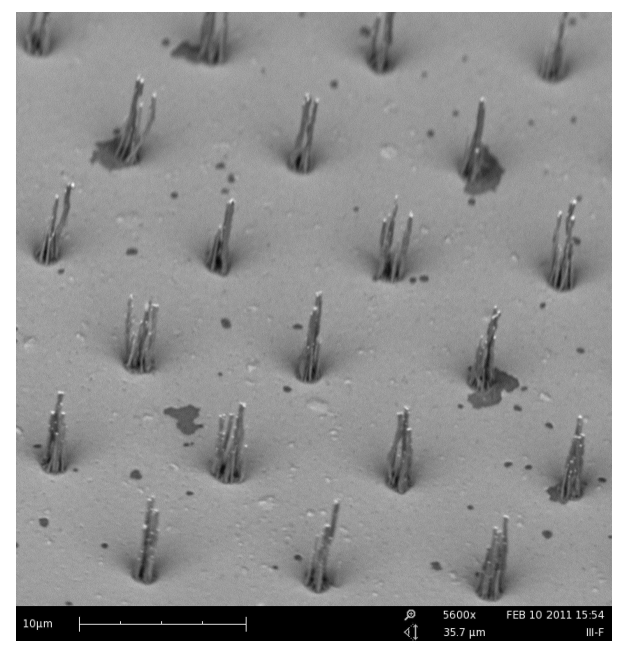

FIG. 1. SEM image of a palladium-coated CNF array (5 nm).

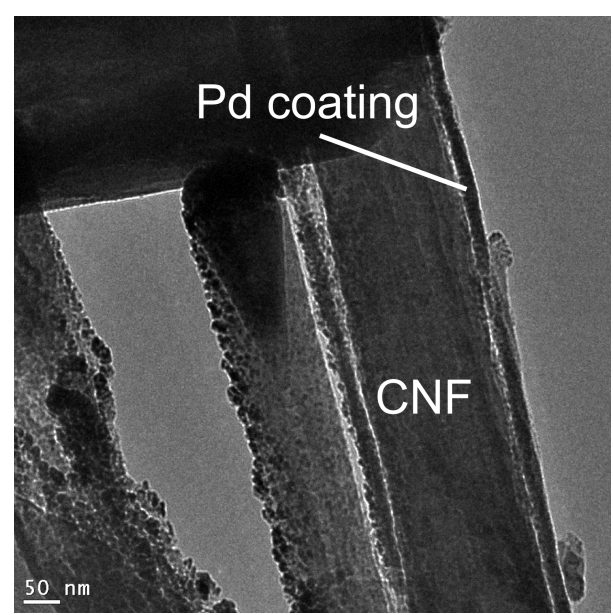

FIG. 2. TEM image of a CNF coated with a $30 \mathrm{~nm}$ layer of palladium.

for neutron production using an external plasma source. The base pressure in the vacuum chamber is $10^{-5} \mathrm{~Pa}$. Deuterium (or hydrogen) gas can be admitted into the chamber using a gas flow controller. Neutrons are measured using a Health Physics Instrument Model 6060 neutron detector. The detector was calibrated using sources of known activity (AmBe and $\mathrm{PuBe}$ ) to enable measurement in units of $\mathrm{n} / \mathrm{s}$. A background level of 0.5 counts $/ \mathrm{min}$ was recorded over several days. For a beam loading experiment of the target the field emitter source was temporarily replaced with a Penning source (Thermo Scientific).

For the electron field emission experiments a second vacuum chamber with a scintillator setup is available. Here the maximum applied voltage is limited to $10 \mathrm{kV}$. However, by moving the electrodes closer together high field strengths can still be achieved. In this setup the scintillator, a Saint-Gobain BC-408, serves as the target electrode and is coated with a thin layer of gold- 


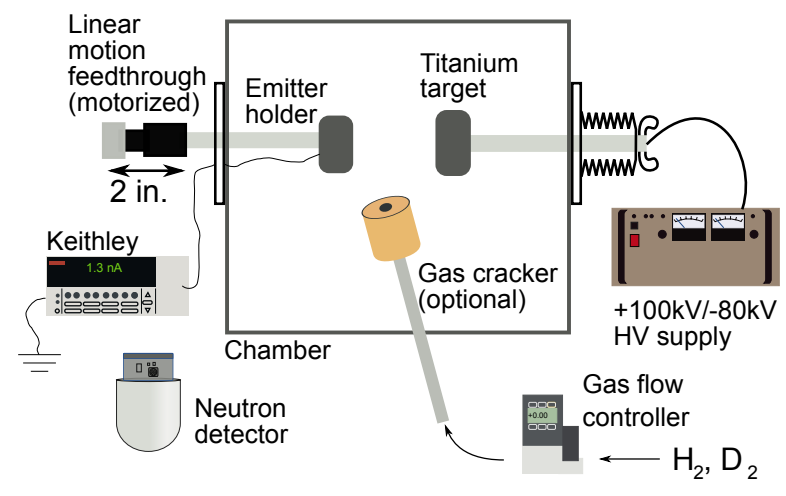

FIG. 3. (Color online) Schematic of the experimental setup for the high-voltage experiments.

palladium to prevent charging. Images of the emission pattern are recorded using a camera.

\section{TARGET}

The titanium target comprises a several millimeters thick titanium layer on a steel body. Titanium is chosen because it can be loaded with deuterium to a high stochiometric ratio of up to $\mathrm{Ti}_{1} \mathrm{D}_{2}$. In order for the fusion reaction to proceed it is important to provide deuterium atoms in the surface layer of the target where the ions will have the highest energy. This can be achieved in two ways: by pre-loading the target or by beam-loading. In the latter case the ion beam itself is used to implant deuterium into the target. Some of the implanted atoms will diffuse to the surface layer where they can then take part in fusion reactions with deuterium ions from the beam. The process of beam-loading depends on the beam conditions, but normally takes about $30 \mathrm{~min}$. A faster way to achieve neutron production is to pre-load the target with deuterium atoms externally, however, it is difficult to achieve a high deuterium content and over time deuterium will diffuse away from the target surface. Indeed, in both cases a high beam-load during the experiment can cause elevated target temperatures which will aid deuterium diffusion and in turn result in a lower neutron output.

The targets used in our experiments were pre-loaded using a plasma process. We use deuterium gas to create a plasma and apply a voltage of $700 \mathrm{~V}$ to the target electrode in order to implant deuterium ions into the target. The amount of deuterium implanted and the loss of deuterium over time was measured for series of titanium samples by elastic recoil detection analysis(ERDA). The samples for this study were pre-loaded at the same time, and hence under the same conditions, and were then measured after different time intervals. The samples were stored under vacuum before analysis. Fig. 4 presents the results, showing a maximum deuterium content of $11 \%$ (assuming a measured deuterium depth pro-

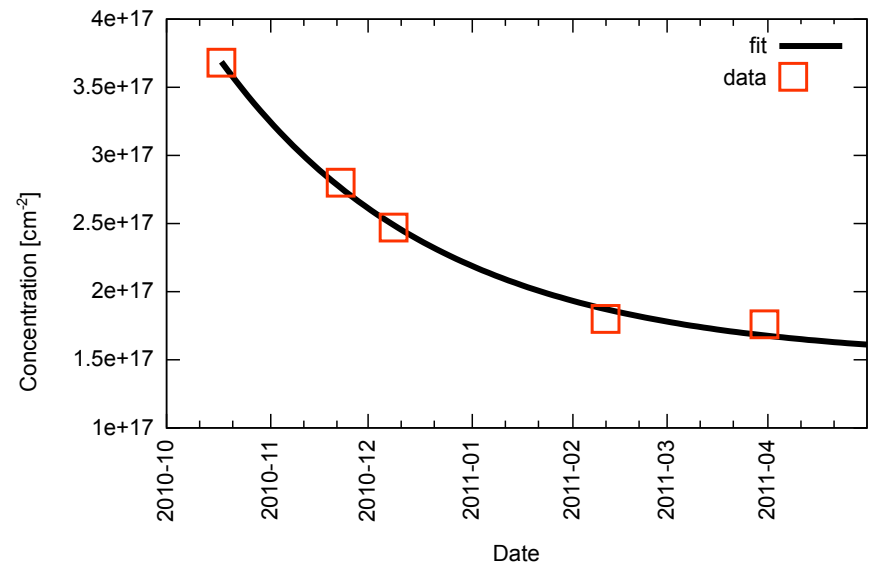

FIG. 4. (Color online) ERDA measurements of deuterated titanium samples.

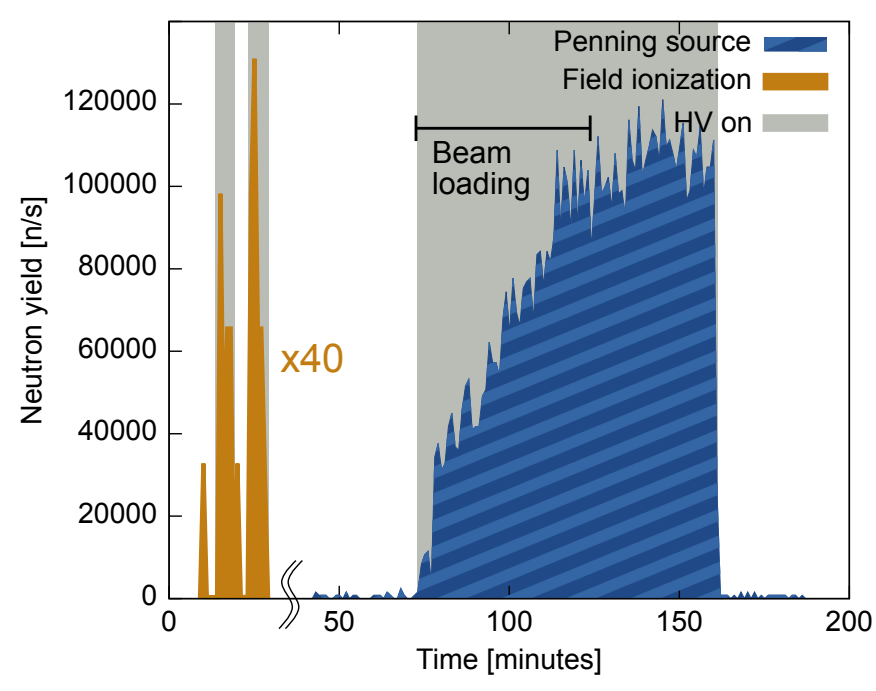

FIG. 5. (Color online) Neutron results from field ionization (left) compared to beam-loading from a Penning source (right). The times when the high voltage was enabled are highlighted.

file of $650 \mathrm{~nm}$ ) and a deuterium loss rate with a half life of 65 days. These data confirm that the pre-loading was successful, albeit resulting in a low deuterium concentration, and that the targets do not loose all of the implanted deuterium in the time frame between pre-loading and the experiment.

We also used a Penning source (Thermo Scientific) to test our setup and to estimate beam loading effects. The beam loading can be clearly seen in Fig. 5 .

The Penning source current was about $4 \mu \mathrm{A}$ which correlates well with our previous neutron yield results (also shown in the picture) and corresponds to a final beam loading of 1:1 of Ti:D, assuming a pre-loading of $11 \%$ as calculated from the ERDA measurements. 


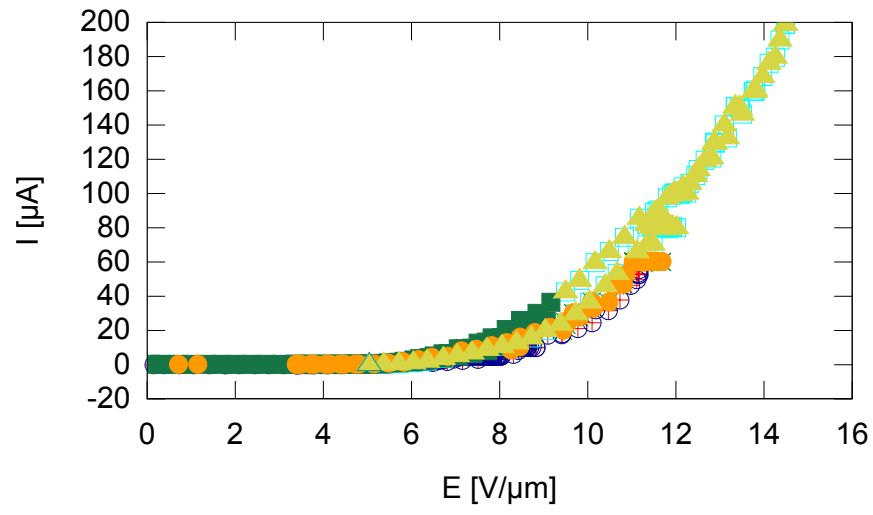

FIG. 6. (Color online) Measurement of current vs. electric field for an uncoated CNF array. The different colors/symbols represent repeated measurements of the same sample.

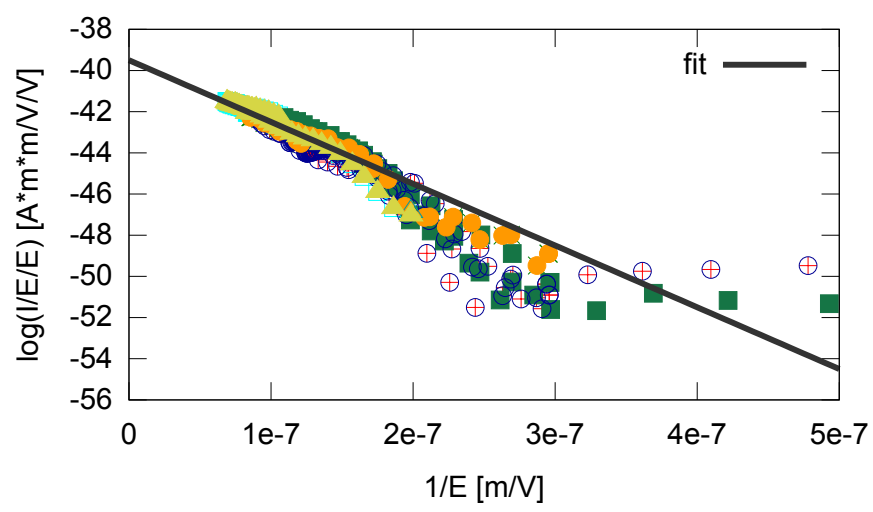

FIG. 7. (Color online) Fowler-Nordheim analysis of the I$\mathrm{V}$ characteristics of an uncoated CNF array. A field enhancement factor of 2400 was measured. The different colors/symbols represent repeated measurements of the same sample.

\section{RESULTS}

I-V curves were recorded for a large number of samples in order to characterize the CNF emitter arrays. Fig. 6 shows the I-V measurements obtained for an uncoated CNF array converted into a plot of current vs. applied electric field. The sample was conditioned by slowly ramping up the current to a few $\mu \mathrm{A}$ and then continuously running the sample for several hours at higher currents. In regular intervals the voltage was ramped down and then up again over a timespan of few minutes in order to gather I-V curves at different times. The various $\mathrm{I}-\mathrm{V}$ curves obtained are shown in the plot. It can be seen that the emission behavior is very stable over time. A Fowler-Nordheim plot, as shown in Fig. 7, shows the linear behavior that is expected for electron field emission. ${ }^{10}$ The field enhancement factor $\gamma$ for this sample is 2400. The Fowler-Nordheim equation used to fit the data is

$$
\log \left(\frac{I}{E^{2}}\right)=\log \left(\frac{c_{a} A \gamma^{2}}{\Phi}\right)-\frac{c_{b} \Phi^{3 / 2}}{\gamma} \frac{1}{E}
$$

where $I(\mathrm{~A})$ is the emitted current, $E(\mathrm{~V} / \mathrm{m})$ is the applied field, $\gamma$ is the field enhancement factor (so that $\gamma E$ is the local field at the tip), $A\left(\mathrm{~m}^{2}\right)$ is the area of the emitter, $\Phi(\mathrm{eV})$ is the work function of the material (4.8 eV was assumed for uncoated CNF), $c_{a}=1.5414 \times 10^{-6} \mathrm{~A} \cdot \mathrm{eV} \cdot \mathrm{V}^{-2}$, and $c_{b}=6.8309 \times$ $10^{9} \mathrm{~V} \cdot \mathrm{m}^{-1} \cdot \mathrm{eV}^{-3 / 2}$.

From the above analysis one can also extract the emitter area. The result is a value much lower than expected indicating that only a small number of tips contribute to the field emission. Since this was a consistent result for a range of samples we have investigated the emission sites further using the scintillator setup. This approach allows direct observation of the emission from different areas of the chip. By testing several samples using the scintillator setup we confirmed that only a small number of tips contribute to the emission current. From simulations of field enhancement assuming wide Gaussian distributions of tip heights and radii we find that only a few tips have large enhancement factors while the majority do not contribute to the emitter current at all. Our simulations also show that a serious issue with our CNF arrays is the large variation in "effective" tip radius, since we in fact grow several tips per island. As a result we are now working on a next generation of tips with a narrow distribution of radii by only growing a single fiber per island. This can be achieved by reducing the size of the nickel catalyst used for the growth process.

During previous experiments using a commercial gas cracker to supply atomic deuterium to the chamber, etching of the CNF emitters was observed. Indeed it is well known that atomic hydrogen can be used to etch carbon. We have started experimenting with different coatings in order to overcome this etching problem and to enable the incorporation of a gas cracker into the source. The SEM and TEM studies discussed earlier showed that palladium leads to a uniform coating and it might be expected that such a coating should be able to protect the fibers from etching effects. Tests using coated emitters showed that almost the same field enhancement factors can be achieved as with uncoated emitters. Relative etching of the coated and uncoated fibers was tested in a hydrogen plasma. Surprisingly, it was found that both sets of emitters survived the plasma treatment with almost no loss in field emitter characteristics. One possible explanation is that the density of atomic hydrogen, which is responsible for the etching effect, differs between the use of the gas cracker and the plasma treatment. Detailed results of the plasma treatment of the field emitter arrays will be published elsewhere. 


\section{SUMMARY AND OUTLOOK}

Arrays of CNF field emitters have been fabricated and analyzed for their field enhancement characteristics with the goal to provide a method to generate ions for a compact and rugged neutron source based on nuclear fusion. Achieving a uniform distribution of field enhancement factors across the CNF arrays has been identified as one of the key challenges in this approach, since a high fraction of emitting tips is required for an ion current large enough to achieve the desired neutron yields. Simulations show that a large variation in tip radii strongly influences the final field enhancement distribution. As a result we have started work on a next generation of field emitter samples with only one CNF per island. This will drastically reduce the variation in tip radii across a sample.

Coated CNF arrays have also been fabricated and characterized, and these results will be reported in more detail in a forthcoming publication. Future work involving surface coatings will include investigations into their use for the catalytic dissociation of molecules to increase the atomic fraction of ions in the beam. This would enable acceleration of the ions to higher energies which is crucial due to the energy dependence of the fusion cross-section.

\section{ACKNOWLEDGMENTS}

The authors would like to thank Kin Man Yu for the ERDA measurements and Frances Allen for the TEM images.

This work was performed under the auspices of the U.S. Department of Energy (DOE), NNSA Office of Nonproliferation Research and Engineering (NA-22) by Lawrence Berkeley National Laboratory under Contract DE-AC02-05CH11231. The TEM and SEM work was performed at NCEM, which is supported by the Office of Science, Office of Basic Energy Sciences of the U.S. Department of Energy (DOE) under Contract No. DEAC02-05CH11231."

${ }^{1}$ Committee on Radiation Source Use and Replacement, Radiation Source Use and Replacement (National Research Council, Washington, D.C, 2008).

${ }^{2}$ L. J. Bond, J. W. Griffin, K. M. Denslow, R. V. Harris, T. Schenkel, A. Persaud, G. E. Dale, A. Sood, J. T. Rutledge, S. Kovaleski, and F. Keel, SEG Exp. Abstr. 29, 518 (2010).

${ }^{3}$ J. Csikai, Handbook of Fast Neutron Generators Volume I (CRC Press, Boca Raton, 1987).

${ }^{4}$ J. Reijonen, AIP Conf. Proc. 1336, 433 (2011).

${ }^{5}$ B. Naranjo, J. K. Gimzewski, and S. Putterman, Nature (London) 434, 1115 (2005).

${ }^{6}$ J. Bonard, N. Weiss, H. Kind, T. Stöckli, L. Forró, K. Kern, and A. Chatelain, Adv. Mater. 13, 184 (2001).

${ }^{7}$ S. H. Jo, Y. Tu, Z. P. Huang, D. L. Carnahan, D. Z. Wang, and Z. F. Ren, Appl. Phys. Lett. 82, 3520 (2003).

${ }^{8}$ A. Persaud, I. Allen, M. R. Dickinson, T. Schenkel, R. Kapadia, K. Takei, and A. Javey, J. Vac. Sci. Technol. B 29, 02B107 (2011).

${ }^{9}$ Y. Zhang, N. W. Franklin, R. J. Chen, and H. Dai, Chem. Phys. Lett. 331, 35 (2000).

${ }^{10}$ R. H. Fowler and L. Nordheim, Proc. R. Soc. London, Ser. A 119, 173 (1928). 


\section{DISCLAIMER}

This document was prepared as an account of work sponsored by the United States Government. While this document is believed to contain correct information, neither the United States Government nor any agency thereof, nor the Regents of the University of California, nor any of their employees, makes any warranty, express or implied, or assumes any legal responsibility for the accuracy, completeness, or usefulness of any information, apparatus, product, or process disclosed, or represents that its use would not infringe privately owned rights. Reference herein to any specific commercial product, process, or service by its trade name, trademark, manufacturer, or otherwise, does not necessarily constitute or imply its endorsement, recommendation, or favoring by the United States Government or any agency thereof, or the Regents of the University of California. The views and opinions of authors expressed herein do not necessarily state or reflect those of the United States Government or any agency thereof or the Regents of the University of California. 